\title{
Primary multifocal extranodal lymphoma of the kidneys, ovaries and bones: utility of FDG PET/CT
}

\author{
Suat-Jin Lu • Mark Ming Loong Tan • \\ Saabry Osmany
}

Received: 5 July 2011/Accepted: 13 September 2011/Published online: 23 September 2011

(C) SIMI 2011

A 25-year-old woman presented with a fever of 1-month duration. There were no specific localizing signs or symptoms. Apart from a raised serum C-reactive protein (108 mg/L, normal $0.2-8.8 \mathrm{mg} / \mathrm{L}$ ) and erythrocyte sedimentation rate $(129 \mathrm{~mm} / \mathrm{h}$, normal $3-15 \mathrm{~mm} / \mathrm{h})$, the blood tests were unremarkable, including a normal full blood count, autoimmune screen, and microbiology tests. The patient completed a course of antibiotics, but the fever persisted. A contrast-enhanced computed tomography (CT) scan of the abdomen and pelvis was performed to evaluate the cause of the prolonged fever. The CT scan showed multiple hypodense lesions in both kidneys (Fig. 1, white arrows), bulkiness of both ovaries (Fig. 1, black arrows), and permeative osteolytic lesions in the bony pelvis (Fig. 1, arrowhead). The differential diagnoses of a malignancy and an infection were entertained. $\mathrm{An}^{18} \mathrm{~F}$-fluorodeoxyglucose positron emission tomography/computed tomography (FDG PET/CT) scan was performed to evaluate the cause of fever, assess the extent of involvement, and determine the most suitable site of biopsy. The FDG PET/CT scan showed multiple hypermetabolic lesions in both kidneys (maximum standardized uptake value, $\mathrm{SUV}_{\max } 16$; Fig. 2, arrows), both ovaries $\left(\mathrm{SUV}_{\max } 24\right.$; Fig. 2, dotted arrow), and multiple bones of the axial and appendicular skeleton, including the calvarium, clavicles, scapulae, multiple ribs bilaterally, multiple vertebrae, bony pelvis, and proximal femora (SUV $_{\max }$ 27; Fig. 2, arrowheads). No nodal disease was detected. A bone marrow aspiration and trephine biopsy were performed, and the results revealed the diagnosis of a

S.-J. Lu (ه) · M. M. L. Tan · S. Osmany Department of Nuclear Medicine and PET, Singapore General Hospital, Outram Road, Singapore 169608, Singapore

e-mail: suat_jin_lu@yahoo.com diffuse large B-cell lymphoma (DLBCL). The imaging and histopathological findings were compatible with a primary multifocal extranodal DLBCL with extensive involvement of the kidneys, ovaries, and bones. After three cycles of R-CHOP chemotherapy (rituximab, cyclophosphamide, doxorubicin, vincristine, and prednisolone), a repeat FDG PET/CT scan showed a complete metabolic resolution of the lesions in the kidneys, ovaries, and bones, indicative of an excellent treatment response (Fig. 3). The patient went on to complete six cycles of chemotherapy, and achieved complete remission.

DLBCL is the largest subtype of non-Hodgkin lymphoma (NHL). Extranodal involvement in the NHL is common with primary extranodal lymphoma accounting for approximately one third of the NHL cases [1, 2]. Extranodal lymphoma can occur in any organ, and can be multifocal involving two or more organs. The most common site of primary extranodal lymphoma is the gastrointestinal tract; the other common sites include the skin, central nervous system and testis [2-4]. Kidneys and ovaries are relatively uncommon sites of primary extranodal lymphoma [2-4]. Concurrent involvement of the kidneys, ovaries and bones in a primary multifocal extranodal lymphoma is an uncommon presentation of a common condition.

Lymphoma is a known cause of a fever of unknown origin (FUO). FDG PET/CT scan has been shown to be useful in the evaluation of FUO [5]. FDG PET/CT scan allows a quick and non-invasive screen of the whole body, and is useful in localizing the source of fever and determining the most accessible site to guide further diagnostic procedures like a biopsy [5]. Hence performing an FDG PET/CT early in the investigation of a prolonged fever may potentially lead to a quicker diagnosis and help to expedite the treatment. 


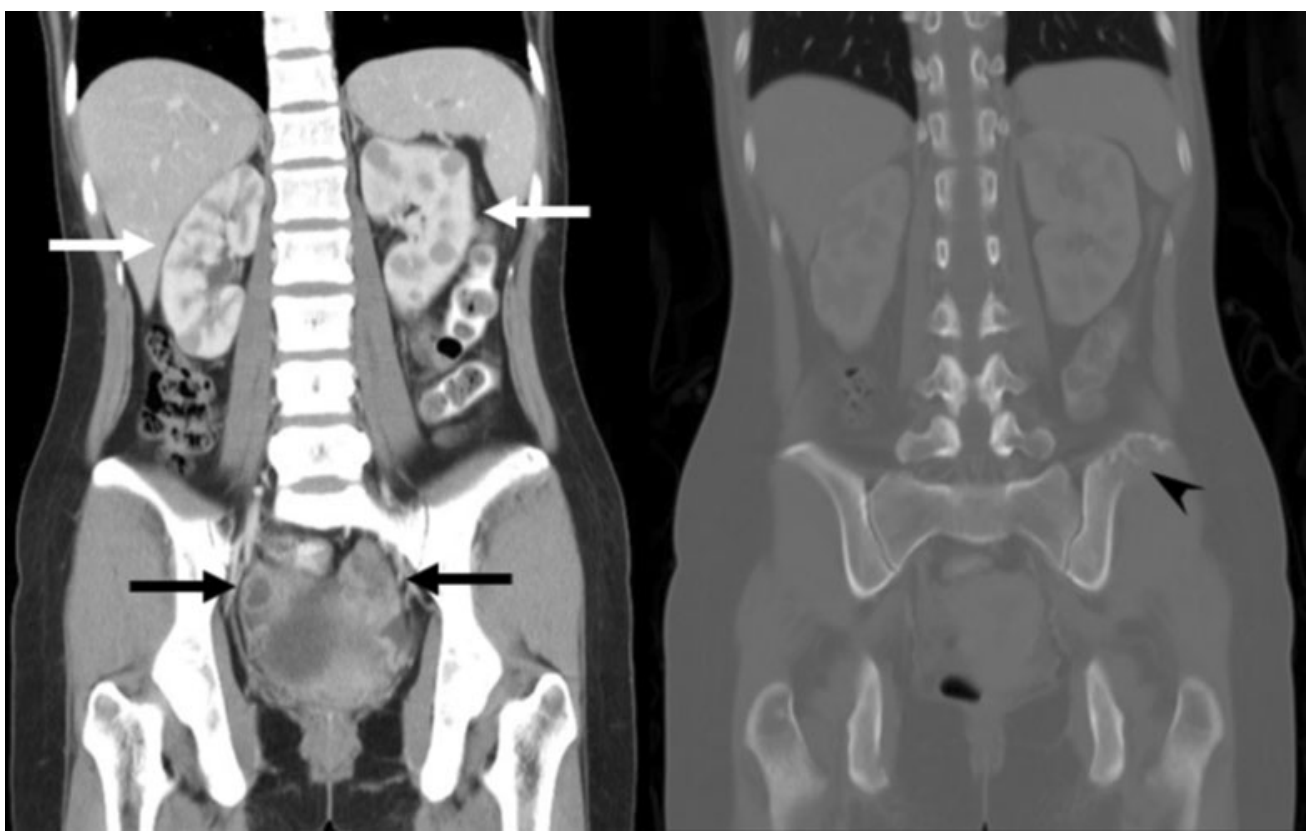

Fig. 1 A contrast-enhanced CT scan showing multiple hypo dense lesions in both kidneys (white arrows), bulkiness of both ovaries (black arrows), and permeative osteolytic lesions in the bony pelvis (arrowhead)
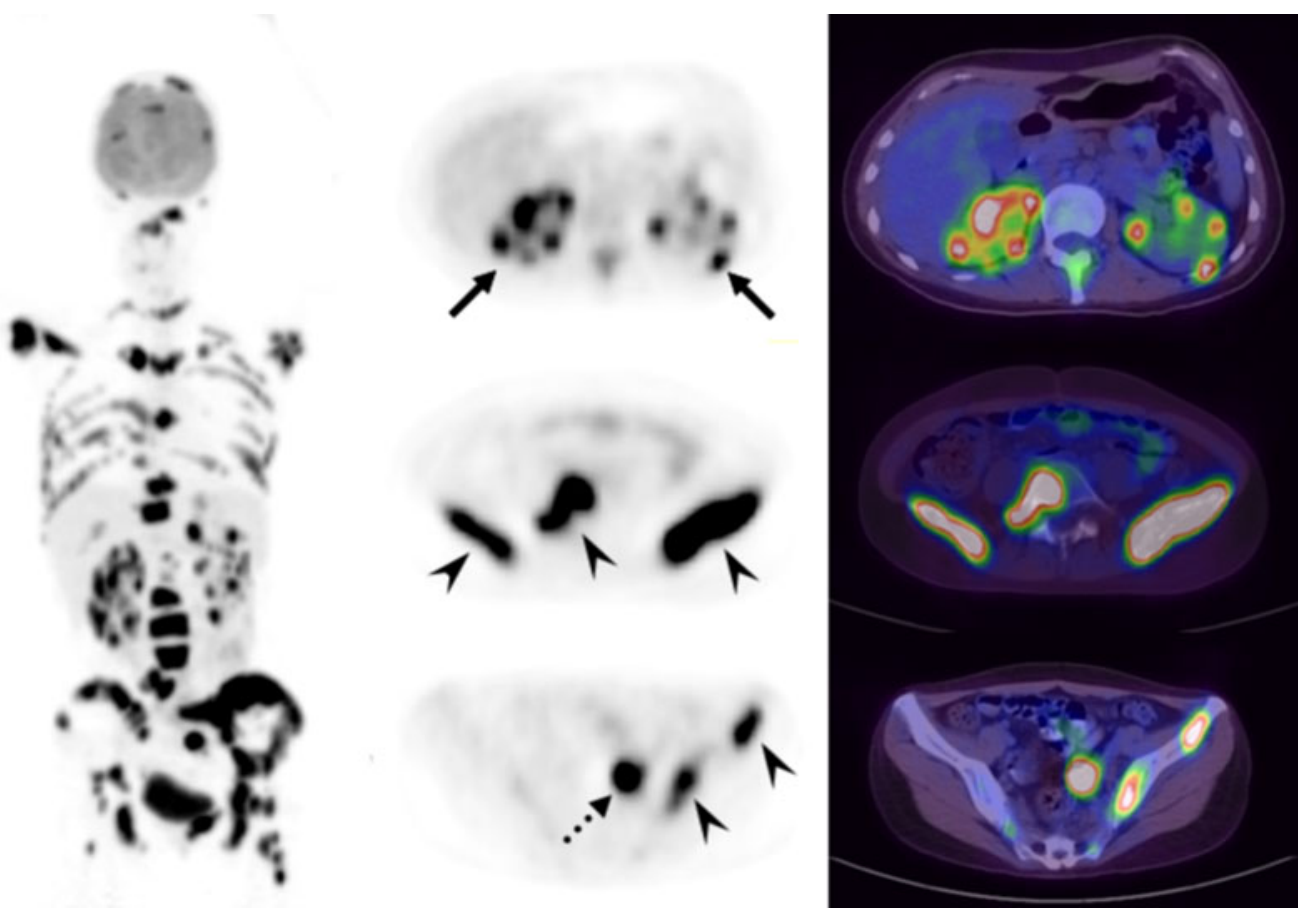

Fig. 2 An FDG PET/CT scan showing multiple hypermetabolic lesions in both kidneys (arrows), both ovaries (dotted arrow), and multiple bones of the axial and appendicular skeleton (arrowheads)

In conclusion, this case demonstrates the imaging findings of an uncommon primary multifocal extranodal DLBCL of the kidneys, ovaries, and bones, and highlights the usefulness of FDG PET/CT scan in evaluating the extent of disease involvement, guiding biopsy, and assessing the treatment response of lymphoma. In addition, 

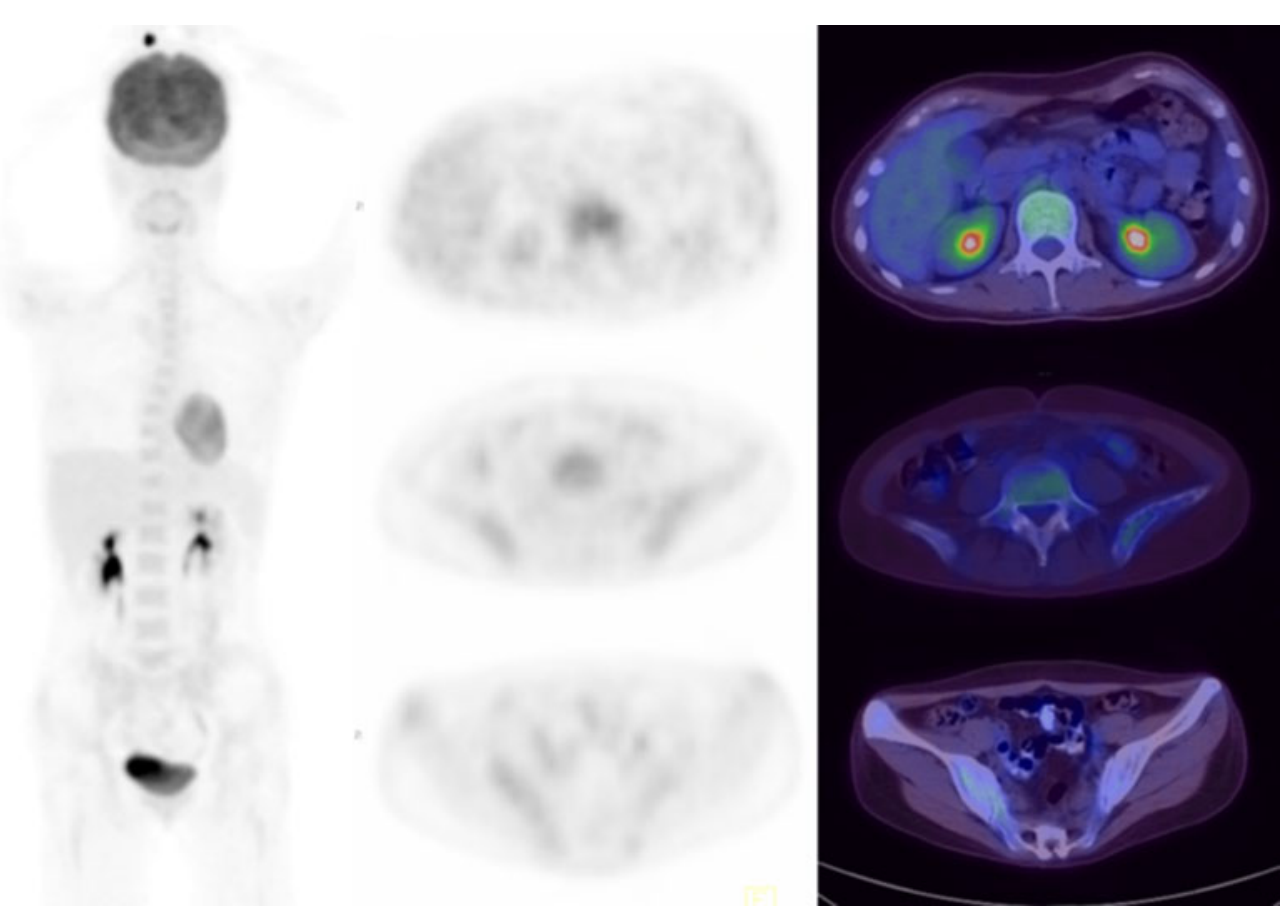

Fig. 3 After three cycles of R-CHOP chemotherapy, a repeat FDG PET/CT scan showed a complete metabolic resolution of all the lesions

this case illustrates the value of performing an FDG PET/ CT scan early in the investigation of a prolonged fever, which may help to expedite diagnosis and treatment.

\section{Conflict of interest None.}

\section{References}

1. López-Guillermo A, Colomo L, Jiménez M et al (2005) Diffuse large B-cell lymphoma: clinical and biological characterization and outcome according to the nodal or extranodal primary origin. J Clin Oncol 23:2797-2804
2. Krol AD, le Cessie S, Snijder S, Kluin-Nelemans JC, Kluin PM, Noordijk EM (2003) Primary extranodal non-Hodgkin's lymphoma (NHL): the impact of alternative definitions tested in the Comprehensive Cancer Centre West population-based NHL registry. Ann Oncol 14:131-139

3. Zucca E, Roggero E, Bertoni F, Cavalli F (1997) Primary extranodal non-Hodgkin's lymphomas. Part 1: gastrointestinal, cutaneous and genitourinary lymphomas. Ann Oncol 8:727-737

4. Zucca E, Roggero E, Bertoni F, Conconi A, Cavalli F (1999) Primary extranodal non-Hodgkin's lymphomas. Part 2: head and neck, central nervous system and other less common sites. Ann Oncol 10:1023-1033

5. Bleeker-Rovers CP, van der Meer JW, Oyen WJ (2009) Fever of unknown origin. Semin Nucl Med 39:81-87 